\title{
A Muon-based Observable to Detect Photons at Ultra-high Energies
}

\author{
N. González ${ }^{a b *}$, F. Sánchez ${ }^{a \dagger}$, M. Roth $^{b}$ and A. Etchegoyen ${ }^{a c}$ \\ a Instituto de Tecnologías en Detección y Astropartículas (CNEA, CONICET, UNSAM), \\ Centro Atómico Constituyentes, Comisión Nacional de Energía Atómica, \\ Av. General Paz 1555 (B1630KNA) San Martín, Buenos Aires, Argentina \\ ${ }^{b}$ Institut für Kernphysik (IKP), Karlsruher Institüt für Technologie, \\ P.O. Box 3640, 76021 Karlsruhe, Germany, \\ ${ }^{c}$ Universidad Tecnológica Nacional, Facultad Regional Buenos Aires, \\ Av. Medrano 951 (C1179AAQ) Ciudad Autónoma de Buenos Aires, Argentina \\ E-mail: nicolas.gonzalez@iteda.cnea.gov.ar
}

\begin{abstract}
The observation of an ultra-high energy photon component in the cosmic radiation is one of the open problems in Astroparticle Physics. The stringent theoretical and experimental upper limits to the photon flux above $100 \mathrm{TeV}$ make the search of a weak photon signal in the vast hadronic cosmic ray background a challenging task. At these energies, photon primaries entering the atmosphere develop an extensive air shower which is driven by electromagnetic processes with a poor muon production. The muon content is one of the most promising observables leading to the discrimination between photons and hadronic cosmic rays. In this article, we define a parameter capable of quantifying the muon component while reducing the fluctuations due to the unknown lateral distribution of muons. We explain the different features of this observable using simulated air showers between 30 and $300 \mathrm{PeV}$. We show that a merit factor of 5 in the separation between photon and proton primaries and a signal efficiency of at least $\sim 92 \%$ while rejecting $99.97 \%$ of the background can be reached considering realistic resolution effects on the shower features.
\end{abstract}

36th International Cosmic Ray Conference -ICRC2019-

July 24th - August 1st, 2019

Madison, WI, U.S.A.

\footnotetext{
* Corresponding author

${ }^{\dagger}$ Speaker.
} 


\section{Introduction}

The detection of photons above PeV energies at Earth is of particular significance, since they are tracers of the highest-energy processes in the Universe. The most violent, but also less understood, astrophysical objects are expected to produce cosmic rays, with an accompanied flux of photons and neutrinos. The simultaneous observation of these particles, the so-called multi-messenger approach, is a key ingredient for discovering the cosmic ray sources themselves.

Very-high energy photons are produced in the vicinity of acceleration regions through cosmic ray interactions with surrounding radiation and matter. In this sense, the H.E.S.S. Collaboration has reported the observation of a gamma-ray spectrum at $\mathrm{TeV}$ energies from the central $70 \mathrm{pc}$ of the Galaxy, implying that the parent protons are being accelerated up to a few PeV [1]. A photon signature at these energies may clarify the feasibility of the supermassive black hole Sagittarius A* at the Galactic center as a cosmic ray source [2]. Indeed, PeV photons have interaction lengths similar to the Milky Way diameter [3], limiting their observability to the local Universe.

Astrophysical neutrinos have been recently discovered by the IceCube Collaboration between $30 \mathrm{TeV}$ and $2 \mathrm{PeV}$ [4]. The hadronic interactions responsible for this neutrino flux would have also produced high-energy photons. However, no direct observation of a photon flux has been confirmed above $81 \mathrm{TeV}$ [5]. The first evidence in favor of the presence of an integral photon flux above tens of $\mathrm{PeV}$ was found in data of the EAS-MSU experiment, although the claim is still under discussion [6]. The non-observation of a diffuse $\mathrm{PeV}$ photon flux supports an extragalactic origin for the IceCube neutrinos. However, the photon surveys were conducted by Observatories located in the geographical northern hemisphere of the Earth (EAS-MSU, KASCADE-Grande [7] and marginally by CASA-MIA [8]) as opposed to most of the neutrino arrival directions. Therefore, conclusions on the non-observation of a $\mathrm{PeV}$ photon flux may be biased, indicating that this problem may still not be completely solved.

Cosmic rays entering the atmosphere interact with the medium nuclei and produce Extensive Air Showers (EAS). The difference in the muon content between photon- and hadron-initiated EAS could be the key to attain the best separation power. In this paper, an observable that combines the density of high-energy muons and their topological distribution to discriminate photon from hadronic primaries is presented. This observable is particularly useful when only a few points in the shower front can be probed for muon detection, so that the lateral distribution of muons cannot be obtained without large uncertainties. We describe the features of the observable based on Monte Carlo air shower simulations with realistic assumptions about experimental features inspired by the surface detector of the Pierre Auger Observatory and its muon detection engineering array $[9,10]$.

\section{Method}

The CORSIKA software was employed to produce the air showers initiated by photons and protons. QGSJetII-04 and FLUKA were chosen as high- and low-energy hadronic-interaction models. The primary energy lies in the range between $E=10^{16.5} \mathrm{eV}$ and $10^{17.5} \mathrm{eV}$ and follows a $E^{-1}$ distribution. The zenith angles are fixed at $\theta=0^{\circ}, 30^{\circ}$ and $40^{\circ}$. The azimuth angle is randomly selected between $0^{\circ}$ and $360^{\circ}$. A thousand unique showers per primary particle and zenith angle were produced. 
Several parameters specific to the Pierre Auger Observatory are taken as a reference in this study $[9,10]$. The list of particles conforming the shower front is given at $878 \mathrm{~g} \cdot \mathrm{cm}^{-2}$ vertical depth, which corresponds to the Auger mean altitude. From the experimental point of view, the quantification of the muon content of the EAS has been historically achieved by arrays of buried detectors, which impose an energy threshold to the observed muons. In this study, an energy threshold of $E_{\text {th }}=1 \mathrm{GeV}$ for vertical muons defines the underground muon content of the EAS, in accordance to the Auger muon detection system.

The lateral density of underground muons on the shower plane $\rho(r)$ may provide enough information to achieve the best separation power between photon (i.e. signal) and proton (i.e. background) primaries. In practice, only a few pieces of information about $\rho(r)$ are available in any measured air shower. In order to recreate this common scenario, the shower footprint is probed at spots separated by a distance $D=433 \mathrm{~m}$ and arranged in a triangular grid. Each spot has a nominal area of $A=60 \mathrm{~m}^{2}$. The core of each simulated EAS is placed randomly in the first Brillouin zone of the hexagonal cell with spacing $D$. The mean number of muons $\overline{n_{i}}$ in a spot $i$ at a distance $r_{i}$ is then $\overline{n_{i}}=\rho\left(r_{i}\right) \times A \cos \theta$. The observed number of muons $n_{i}$ is randomly selected from a Poisson distribution with mean $\overline{n_{i}}$. The process is repeated ten times for each shower, probing different regions of the shower footprint. Each realization is called an event.

The features of the EAS can be accurately estimated within resolutions imposed by the employed array of detectors. To resemble a realistic experimental scenario, smearing and efficiency effects are applied on the event energy $E$, the distance $r_{i}$ between the spots and the shower axis and each observed muon density $\rho_{i}$. An energy resolution of $\triangle E=16 \%$ [9] and a shower core position resolution of $\Delta r=20 \mathrm{~m}$ [11] are considered as representative values. Each spot is treated as a muon counter with a systematic uncertainty $\Delta \rho=11.2 \%$ on the observed muon density $\rho_{i}$ [12] and a penalization factor $\varepsilon=90 \%$ accounting for possible inefficiencies. Thus, $\rho_{i}$ is redefined as $\widetilde{\rho}_{i}=\rho_{i} \times \varepsilon$. Lastly, each event is re-weighted with a factor proportional to $E^{-3.3}$, in consonance with the observed cosmic ray spectrum [13].

The observable $M_{b}$ combines the density of muons and their topological distribution as defined in Eq. 2.1, inspired by the observable $S_{b}$ designed with signals measured by water-Cherenkov detectors [14]. The muon densities are normalized by the expected muon density for a proton primary $\rho_{\text {ref }}(E, \theta)$ at a reference distance $r_{\text {ref }}=250 \mathrm{~m}$, where the fluctuations of the muon density are minimal for the energy range under study. The index $b$ is a free parameter that must be selected as to maximize the separation between primaries.

$$
M_{b}(E, \theta)=\log _{10}\left(1+\sum_{i}\left(\frac{\rho_{i}}{\rho_{\mathrm{ref}}}\right) \times\left(\frac{r_{i}}{r_{\mathrm{ref}}}\right)^{b}\right)
$$

Since the hadronic background is hugely dominant over any photon component in the cosmic ray flux, the most important feature of $M_{b}$ is the background rejection, i.e. the proportion of filtered proton events to the corresponding total by a straight-forward cut in the observable phase space. Similarly, the ratio between the photon events surviving the cut and the total number of photon events is defined as the signal efficiency. A complete background rejection can be thought of as a statistical fluctuation from the general scenario in which the cosmic ray flux could have produced a mean number $N_{\mathrm{br}}$ of background events surviving the cut. The probability $P\left(N_{\mathrm{obs}} \mid N_{\mathrm{br}}\right)$ to observe 
$N_{\text {obs }}=0$ events for an expected average $N_{\text {br }}$ is given by the Poisson statistics. By asking for $P<$ $0.05, N_{\mathrm{br}}$ is estimated at a $95 \%$ confidence level (CL). For the particular case of $N_{\mathrm{obs}}=0, N_{\mathrm{br}}^{95 \%} \simeq 3$. Therefore, the background rejection at $95 \% \mathrm{CL}$ is redefined as $N-3 / N$, where $N$ is the number of available background events in the subset under study. The associated signal efficiency is thus the maximum possible value assuring $N_{\mathrm{obs}}=0$. These conservative numbers are the benchmark rejection and efficiency of the procedure.

\section{Results}

The optimal value $b_{\text {opt }}$ should be chosen according to the merit factor ${ }^{1} \eta$ between photon and proton events, displayed in Fig. 1, left, for events with energies between $E=10^{16.5} \mathrm{eV}$ and $10^{16.7} \mathrm{eV}$ and fixed zenith angle of $\theta=0^{\circ}$. In view of the layout used in this study, four scenarios are considered: i) the observed muon density at the nearest probing spot is used (gray dashed), ii) adding the information from the first crown of spots around the nearest one (dark blue), iii) also adding the information from the second crown (blue) and iv) summing the contributions of the muon densities up to the third crown (light blue).

The information solely provided by the nearest spot comprises a merit factor of $(3.03 \pm 0.08)^{3}$ for $b_{\text {opt }}=1.5$. The merit factor is increased to $(5.04 \pm 0.15)$ when accounting for the observed muon densities in the first crown of spots (scenario ii) for $b_{\mathrm{opt}}=1$. A similar merit factor an be attained in scenario iii and iv respectively. Indeed, as the distances at which the shower front is probed increase, the observed muon densities reach the resolution limit given by the nominal area. At this point, no relevant information towards discrimination is added to $M_{b}$ and the separation reaches a saturation level. Since the discrimination between signal and background does not improve considerably in scenario iii and iv wrt scenario ii, $M_{b}$ is calculated with the nearest spot and its surrounding first crown for the rest of this article.

The essential concepts behind the procedure do not depend on the primary energy and zenith angle. Indeed, Fig. 1, right, shows the merit factor in terms of $b$ for different energy bins and $\theta=0^{\circ}$. The choice of $b_{\mathrm{opt}}=1$ is nearly optimal for the entire energy range of interest. The specific choice for $b_{\text {opt }}$ holds also in the non-vertical case, thanks to the weak zenith dependence of $M_{b}$.

An increasing merit factor does not necessarily point towards a better primary discrimination in a shower-to-shower basis. To obtain a reliable estimation of the background rejection and the signal efficiency, proxy $M_{1}$ distributions are built with events corresponding to single core tosses, resulting in ten proton and photon independent sets for each energy and zenith angle bins. The number of proton events verifying $M_{1}^{p}>M_{1}^{\text {cut }}$ define the background rejection for running values of $M_{1}^{\text {cut }}$. Likewise, the number of photon events verifying $M_{1}^{\gamma}<M_{1}^{\text {cut }}$ define the signal efficiency. The background rejection in terms of the signal efficiency profile is thus the average between the

\footnotetext{
${ }^{1} \mathrm{~A}$ modified version of the merit factor is used to take into account the asymmetries of the $M_{b}$ distributions [14]. Medians are used instead of the means and quantiles at $84 \%$ and $16 \%$ define the dispersion of the distribution.

${ }^{2}$ This is motivated by the fact that the atmospheric absorption of the secondary particles prevents the detection of low-energy inclined air showers.

${ }^{3}$ The average values and the $1 \sigma$ interval, quoted as errors, are obtained through 100 independent runs of the event generation procedure described in Sec. 2.
} 

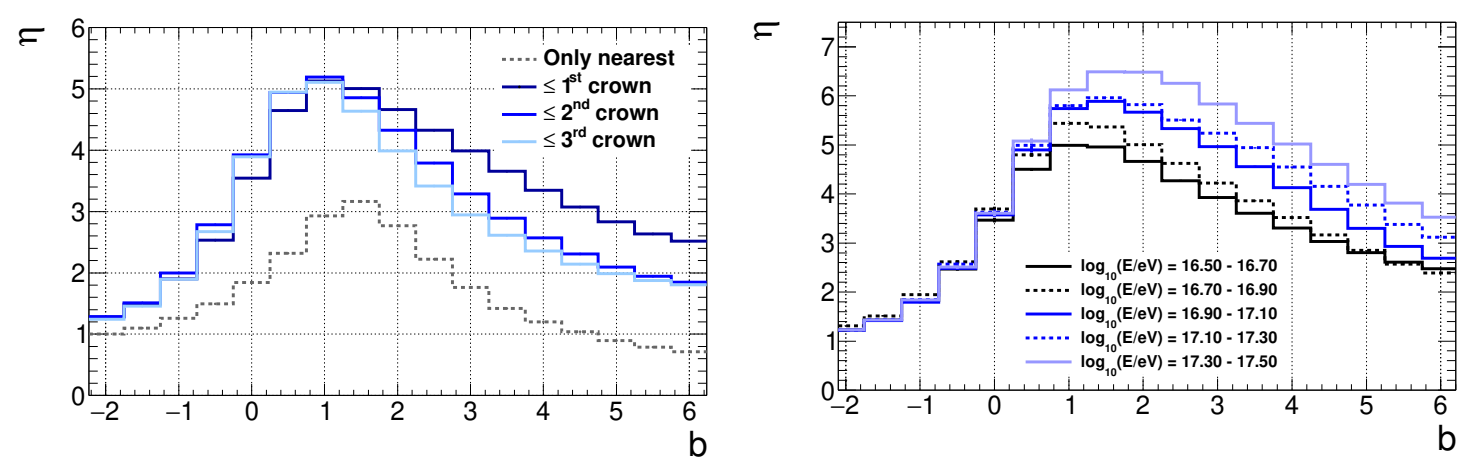

Figure 1: Left: The merit factor $\eta$ of the observable $M_{b}$ in terms of $b$. Different scenarios for the $M_{b}$ calculation are explored (see text for descriptions). The events have energies between $E=10^{16.5} \mathrm{eV}$ and $10^{16.7} \mathrm{eV}$. The zenith angle is fixed at $\theta=0^{\circ}$. Right: The merit factor in terms of the index $b$ between vertical photon and proton events. Different colors represent the energy ranges.

ten realizations. The confidence band is delimited by the minimum and maximum background rejection for a given signal efficiency among all realizations.

The separation power expressed through the background rejection profiles does not follow a clear trend in terms of the primary energy as shown in the example of Fig. 2 due to a two-fold effect. On the one hand, the early drops of some of the profiles embody the presence of a handful of proton events with a poorly-probed muon footprint with the consequent understimated $M_{1}$. On the other hand, a population of photon events generating $M_{1}$ values typically assigned to background events is visible at all primary energies and zenith angles. Both effects share physical origins, as discussed in Sec. 4.

A benchmark background rejection of 99.95\% is reached with an associated median signal efficiency of at least $\sim 95 \%$ for the events between $E=10^{16.5} \mathrm{eV}$ and $10^{16.7} \mathrm{eV}$,

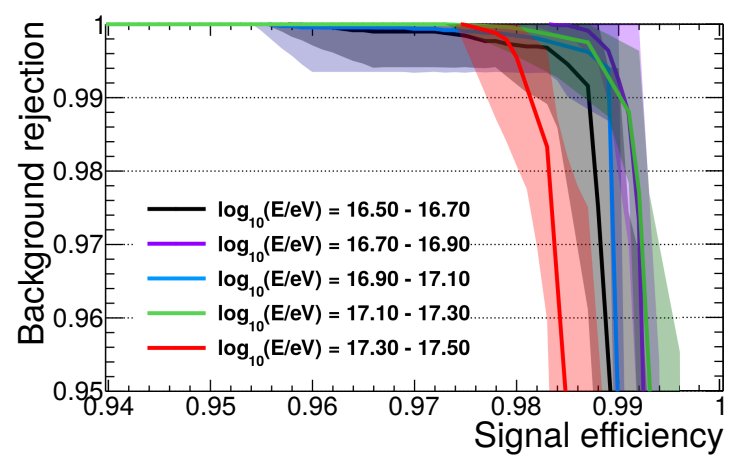

Figure 2: The background rejection in terms of the signal efficiency for different energy bins and a fixed zenith angle of $\theta=30^{\circ}$. The profiles are obtained as the average of the ten produced events per shower while the contour regions represent the areas between the minimum and maximum background rejection in each signal efficiency bin. as deduced from Tab. 1. The underlying limited resolution due to the low statistics appears as a decrease of the accessible benchmark rejection as the primary energy increases. Complementarily, the signal efficiency displays a stable behaviour across the energy range under study, interpreted as a constant discrimination power regardless of the statistics in each energy bin. Given the 10000 available background events for each zenith angle, the energy-integrated benchmark rejection is fixed by construction at $99.97 \%$. The associated median benchmark efficiencies are $\left(95.1 \pm_{2.1}^{1.4}\right) \%$, $\left(92.0 \pm_{5.7}^{1.3}\right) \%$ and $\left(94.0 \pm_{5.0}^{2.2}\right) \%$ for a fixed zenith angle of $\theta=0^{\circ}, 30^{\circ}$ and $40^{\circ}$, respectively ${ }^{4}$.

\footnotetext{
${ }^{4}$ The uncertainties represent the $16 \%$ and $84 \%$ quantiles of the distributions after 100 independent realizations
} 


\begin{tabular}{ccccccc}
\hline$E(\mathrm{eV})$ & \multicolumn{3}{c}{ Benchmark rejection } & \multicolumn{3}{c}{ Benchmark efficiency } \\
\hline \hline $10^{16.5}-10^{16.7}$ & $99.95 \%$ & $99.95 \%$ & $99.95 \%$ & $\left(97.4 \pm_{0.8}^{0.6}\right) \%$ & $\left(94.9 \pm_{5.9}^{2.4}\right) \%$ & $\left(96.3 \pm_{1.7}^{1.0}\right) \%$ \\
\hline $10^{16.7}-10^{16.9}$ & $99.87 \%$ & $99.89 \%$ & $99.87 \%$ & $\left(96.5 \pm_{1.7}^{1.2}\right) \%$ & $\left(95.6 \pm_{3.4}^{2.5}\right) \%$ & $\left(97.9 \pm_{2.0}^{0.8}\right) \%$ \\
\hline $10^{16.9}-10^{17.1}$ & $99.63 \%$ & $99.62 \%$ & $99.63 \%$ & $\left(97.0 \pm_{2.6}^{1.2}\right) \%$ & $\left(97.3 \pm_{4.4}^{1.1}\right) \%$ & $\left(97.5 \pm_{2.8}^{0.9}\right) \%$ \\
\hline $10^{17.1}-10^{17.3}$ & $98.99 \%$ & $98.83 \%$ & $98.91 \%$ & $\left(98.4 \pm_{0.7}^{0.6}\right) \%$ & $\left(97.9 \pm_{0.6}^{0.5}\right) \%$ & $\left(97.2 \pm_{1.7}^{0.8}\right) \%$ \\
\hline $10^{17.3}-10^{17.5}$ & $96.67 \%$ & $96.61 \%$ & $96.82 \%$ & $\left(97.0 \pm_{1.0}^{0.8}\right) \%$ & $\left(97.5 \pm_{0.6}^{0.8}\right) \%$ & $\left(98.0 \pm_{1.0}^{0.6}\right) \%$ \\
\hline
\end{tabular}

Table 1: The mean benchmark rejection and the median benchmark efficiency considering events reweighted by a power-law spectrum $E^{-3.3}$ ) after 100 independent realizations of the event generation procedure in each energy bin. The uncertainties in the benchmark efficiency represent the $16 \%$ and $84 \%$ quantiles. The three columns for each quantity correspond to the fixed zenith angles of $\theta=0^{\circ}, 30^{\circ}$ and $40^{\circ}$.

\section{Discussion}

The muon content of the EAS, quantified by the observable $M_{b}$, provides an energy-integrated benchmark efficiency of at least $\sim 92 \%$ and a guaranteed background rejection level of $99.97 \%$, only constrained by the limited statistics. The topological aspect of $M_{b}$ is mainly contained in the index $b$ : negative (positive) values would give more importance to regions of the shower front close to (far away from) the shower axis ${ }^{5}$. Therefore, the choice of $b_{\text {opt }}=1$ can be thought of as a minimization of the observable fluctuations, considering that larger fluctuations in the muon number are expected closer to the core, due to the inherent variations of the point of first interaction between the primary particle and the atmosphere, and for increasing distances, due to the smaller muon density.

A small fraction of the photon events composing the long tail towards proton-like regime is readily observed in Fig. 3, left, which can be understood as an effect of the early stages of the shower development: if one of the first interactions is photonuclear, the subsequent development would be more similar to a proton shower. In addition, there is a subset of proton samples composing a resilient tail towards the photon distributions. These proton showers have several features similar to the photon-initiated EAS, such as the extremely poor muonic content and the delayed longitudinal profile. This may be related to a high-energy leading $\pi^{0}$ in the first stages of the shower development carrying away a considerable fraction of the primary energy [15], whose decay triggers an electromagnetic sub-shower with the related photon-like characteristics. In this regard, the atmospheric depth of the maximum shower development $X_{\max }$ is displayed in Fig. 3, right, connected to $M_{1}$ for both primaries. The impact of these muon-poor events could be reduced if a hybrid $X_{\max }-M_{1}$ approach is developed, exploiting the distinctive aspects of the photon-initiated EAS.

The discrimination procedure presented in this article is sustained on robust foundations. The mass composition of the background particles has been considered as pure-proton to develop the

\footnotetext{
${ }^{5}$ The hadronic primaries have a larger probability of producing a non-null muon signature in spots at larger distances than the photon ones. Thus, the relative weight in $M_{b}$ of spots at distances similar to $r_{\text {ref }}=250 \mathrm{~m}$ is enhanced wrt closer spots with a selection of a positive $b_{\text {opt }}$.
} 

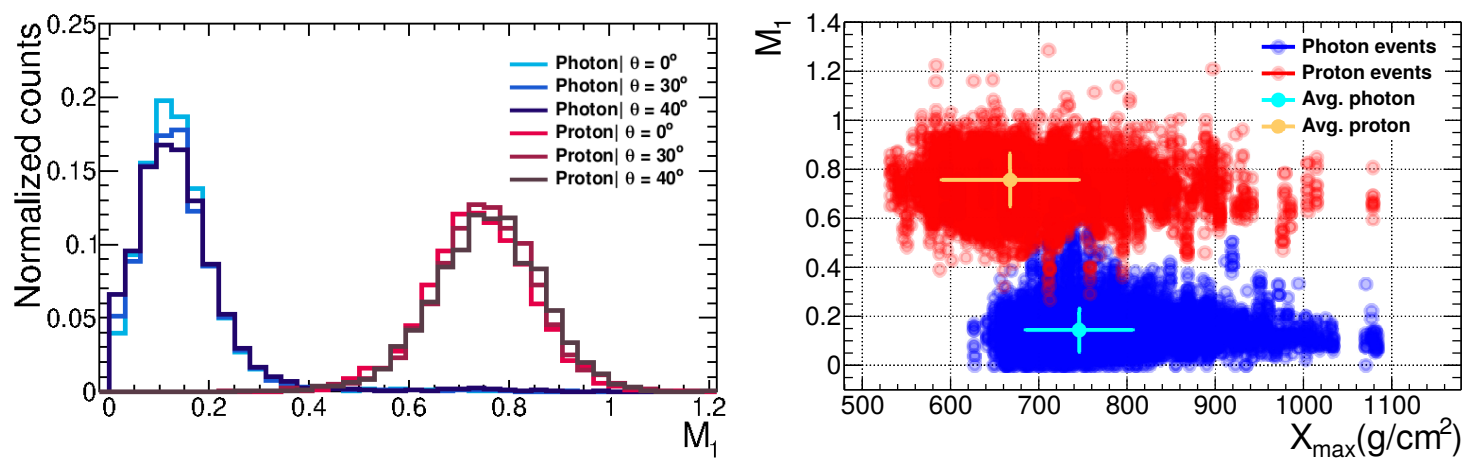

Figure 3: Left: The $M_{1}$ distributions for photon (in blue scale) and proton (in red scale) primaries. In both panels, all events are considered with the corresponding energy-based weight. The histograms are normalized to the number of entries.Right: $M_{1}$ in terms of the atmospheric depth of maximum shower development $X_{\max }$. The events produced by photon (proton) primaries are represented by blue (red) markers. All events with $\theta=30^{\circ}$ are plotted together, indistinctively of the primary energy. The weighted mean value of the observables for both populations are represented by light circle markers, with the $68 \%$ confidence interval as error bars.

procedure in a conservative scenario. The cosmic ray flux may well have a non-negligible presence of heavier nuclei [16]. Therefore, a larger muon content may be present in data, enhancing the benchmark efficiency. Additionally, there is a general agreement that all the hadronic models underestimate the muon content of the EAS [17]. The muon deficit in simulations could be as large as $\sim 50 \%$ as recently reported above $10^{17.5} \mathrm{eV}$ by employing the Auger muon detection system [12]. Thus, the discrimination power of observables directly linked to the number of muons may be underestimated.

The calculation of $M_{b}$ is sufficiently flexible as to easily be adapted to any specific array geometry. In this work, the chosen array spacing and the primary energies of interest pointed out the need of considering the nearest spot and the first crown around it while choosing $b=1$ in order to optimize the separation power. However, those two aspects may be varied accordingly depending on the case under study. Similarly, the threshold for the muon detection (here chosen as $1 \mathrm{GeV}$ ) plays a major role in the cosmic ray mass composition studies [18] and may be adjusted consonantly to the technical specifications of the available detection system.

To conclude, the observable $M_{b}$ offers the possibility of a straight-forward photon search procedure by using the information of the muon density at a few points of the shower front, relying on the reconstruction of the primary particle and EAS properties known a priori from a different technique. A signal efficiency of at least $\sim 92 \%$ can be attained at a $99.97 \%$ background rejection in the energy range between $E=10^{16.5} \mathrm{eV}$ and $10^{17.5} \mathrm{eV}$ for a $E^{-3.3}$ spectrum and after applying realistic detector-like smearing effects in the primary energy, shower core position and muon counting. Remarkably, in view of the cosmic ray composition at the energies of interest for this study being, in average, heavier than proton and of the current hadronic interaction models underestimating the muon content in EAS, the performance of $M_{b}$ when applied to data might be above the results presented in this article. 


\section{References}

[1] The HESS Collaboration. Acceleration of petaelectronvolt protons in the Galactic Centre. Nature, 531:476-479, March 2016. [astro-ph.HE/1603.07730].

[2] F. A. Aharonian. Gamma rays from supernova remnants. Astropart. Phys, 43:71-80, March 2013.

[3] M. Settimo and M. De Domenico. Propagation of extragalactic photons at ultra-high energy with the EleCa code. Astropart. Phys., 62:92-99, March 2015.

[4] The IceCube Collaboration. Observation of Astrophysical Neutrinos in Six Years of IceCube Data. In $35^{\text {th }}$ International Cosmic Ray Conference, volume 301, August 2017.

[5] The HESS Collaboration. Primary particle acceleration above $100 \mathrm{TeV}$ in the shell-type Supernova Remnant RX J1713.7-3946 with deep H.E.S.S. observations. Astron. Astrophys., 464:235-234, November 2007.

[6] Y. Formin et al. Constraints on the flux of $10^{16}-10^{17.5} \mathrm{eV}$ cosmic photons from the EAS-MSU muon data. Phys. Rev. D, 95(12), June 2017.

[7] The KASCADE-Grande Collaboration. KASCADE-Grande Limits on the Isotropic Diffuse Gamma-Ray Flux between $100 \mathrm{TeV}$ and 1 EeV. Astrophys. J., 848(1), October 2017.

[8] M. C. Chantell et al. Limits on the Isotropic Diffuse Flux of Ultrahigh Energy Gamma Radiation. Phys. Rev. Lett., 79(1805), September 1997.

[9] The Pierre Auger Collaboration. The Pierre Auger Cosmic Ray Observatory. Nucl. Instr. Meth., 798:172-213, October 2015.

[10] The Pierre Auger Collaboration. Prototype muon detectors for the AMIGA component of the Pierre Auger Observatory. J. Instrum., 11, February 2016.

[11] K. Rawlins for the IceCube Collaboration. Cosmic ray spectrum and composition from three years of IceTop and IceCube. J. Phys. Conf. Ser., 718(052033), September 2016.

[12] S. Müller for the Pierre Auger Collaboration. Direct measurement of the muon density in air showers with the Pierre Auger Observatory. In EPJ Web of Conferences, editor, International Symposium on Ultra High Energy Cosmic Rays, volume 210, May 2019.

[13] The Telescope Array Collaboration. The Cosmic-Ray Energy Spectrum between 2 PeV and 2 EeV Observed with the TALE detector in monocular mode. Astrophys. J., 865(1), September 2018.

[14] G. Ros et al. A new composition-sensitive parameter for Ultra-High Energy Cosmic Rays. Astropart. Phys., 35(3):140, October 2011.

[15] L. Lu. A search for photons of energy above $6 \times 10^{18} \mathrm{eV}$ using data from the Water-Cherenkov detectors of the Pierre Auger Observatory. PhD thesis, University of Leeds, 2014.

[16] J. Bellido for the Pierre Auger Collaboration. Depth of maximum of air-shower profiles at the Pierre Auger Observatory Measurements above $10^{17.2} \mathrm{eV}$ and Composition Implications. In Proceedings of Science, editor, 35 ${ }^{\text {th }}$ International Cosmic Ray Conference, volume 301, July 2017.

[17] T. Pierog. Air Shower Simulation with a New Generation of post-LHC Hadronic Interaction Models in CORSIKA. In Proceedings of Science, editor, $35^{\text {th }}$ International Cosmic Ray Conference, volume 301, July 2017.

[18] S. Müller et al. Impact of muon detection thresholds on the separability of primary cosmic rays. Astropart. Phys., 97:174-185, January 2018. 